\title{
Implantable Microelectrodes with New Electro-Conductive Materials for Recording Sympathetic Neural Discharge
}

\author{
Kanji MATSUKAWA, Hidehiko KOMINE, Hirotsugu TSUCHIMOCHI, Jun MuRATA, \\ Yoshiharu YONEZAWA*, Kazuhiko KONDO ${ }^{\dagger}$, and Yasuo SEKI ${ }^{\dagger}$ \\ Department of Physiology, Institute of Health Sciences, Hiroshima University Faculty of Medicine, Hiroshima, 734-8551 \\ Japan; * Department of Electrical and Digital Systems Engineering, Hiroshima Institute of Technology, \\ Hiroshima, 731-5193 Japan; and † Nippon Cable System, Inc., Takarazuka, 665-0845 Japan
}

\begin{abstract}
We have developed two new types of bipolar cuff microelectrodes (volume size, 2-3 $\mathrm{mm}^{3}$ ) using electro-conductive rubber or water-absorbent polymer, either of which can be applied to measure sympathetic nerve activity in small animals. A renal nerve bundle of an anesthetized rat was inserted into the center hole of
\end{abstract}

the electrode (diameter, $0.15 \mathrm{~mm}$ ) through a slit and had good contact with the electrodes. Renal sympathetic nerve activity, which was verified by sympathetic ganglionic blockade, could be recorded using either type of implantable cuff electrode. [Japanese Journal of Physiology, 53, 61-64, 2003]

Key words: implantable electrode, sympathetic nerve activity, cardiovascular control.

$\mathrm{I}_{\mathrm{n}}$ trol of the cardiovascular system, it is important to directly measure multiple sympathetic outflows to various vascular beds and analyze the long-term changes in sympathetic outflow following a given intervention such as drug infusion and denervation surgery, because the sympathetic nervous system achieves differential and integrative control of blood flows to different organs and their physiological functions. Original mass activity of sympathetic postganglionic nerve fibers has been measured by implanting a bipolar cuffelectrode on a sympathetic nerve bundle or by carefully winding a pair of bipolar wire-electrodes onto the nerve bundle. As electro-conductive materials for the electrodes, biomaterial substances of collagen or metal-wires (silver or stainless steel) have been used so far [1-4]. Using conventional electrodes, renal sympathetic nerve activity has been successfully recorded in different species including conscious cats $[1-3,5]$, rabbits $[6,7]$, rats $[4,8]$, and dogs $[9,10]$. However, sympathetic outflow to any other organ but the kidneys has not been measured, except cardiac sympathetic nerve activity in cats [11-13] and sheep [14], and lumbar sympathetic nerve activity in rats
[15]. The difficulty of recording sympathetic nerve activity was not only due to the surgical limitation of isolating nerve bundles, but also due to substantial technical problems with conventional recording electrodes: First, conventional electrodes are too large to apply to small nerve bundles and might cause mechanical damage to the nerve. Second, it is difficult to implant a bipolar cuff-electrode on a nerve bundle or to wind a pair of bipolar wire-electrodes onto a nerve bundle, so skillful surgical technique becomes a prerequisite. Third, it is impossible to measure sympathetic nerve activity for more than 7-10 d after implantation surgery. In addition, the magnitude and signal-to-noise ratio of sympathetic nerve activity deteriorate over time, even though sympathetic nerve activity is obviously identified. Therefore, the invention of a novel miniature electrode that can be applied to a small nerve bundle in free moving animals is needed for stable, long-term, and simultaneous recording of sympathetic neural discharges to various vascular beds.

In the present study, we developed two kinds of bipolar cuff microelectrodes made with new materials, electro-conductive rubber or water-absorbent polymer, 

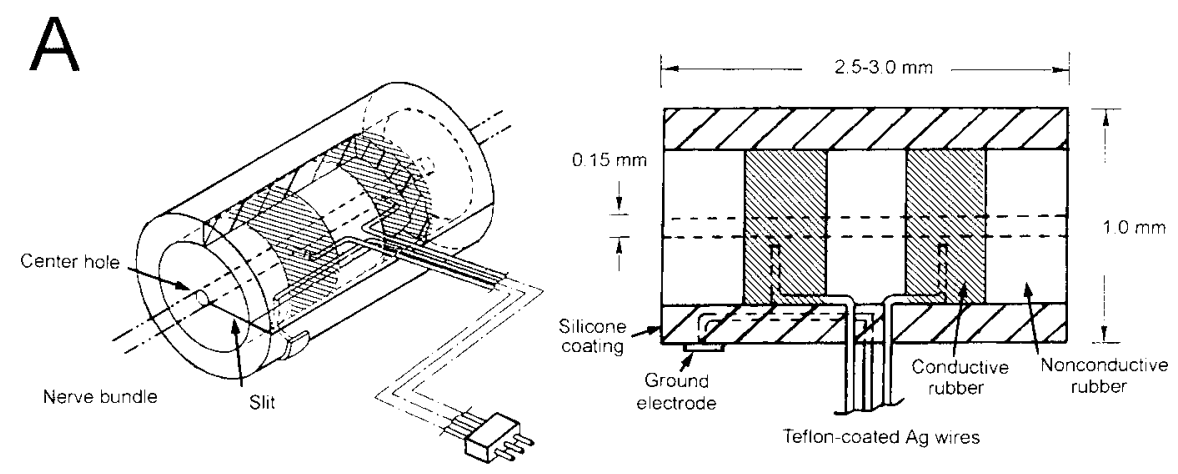

B
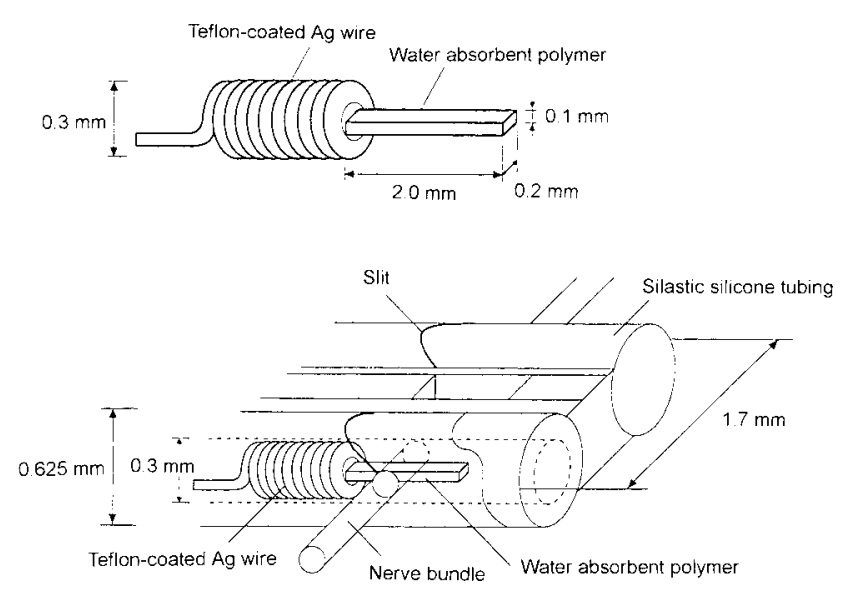

Fig. 1. Schematic configurations of novel implantable microelectrodes using electro-conductive rubber (A) and water-absorbent polymer (B). as shown in Fig. 1. A preliminary report of this work has been published [16]. Figure 1A illustrates the configuration of the electro-conductive rubber (ECR) electrode. The ECR electrode consists of five layers of cylindrical rubber structures arrayed in series. The cylindrical rubber structures are made of conductors and nonconductors. A pair of Teflon-coated silver wires (diameter, $0.1 \mathrm{~mm}$ ) was firmly fixed to the ECR. The diameter of the ECR electrode is $1 \mathrm{~mm}$ and its length is $2.5-3 \mathrm{~mm}$; accordingly, the volume of the electrode is $2.0-2.4 \mathrm{~mm}^{3}$. The outer surface of the electrode is coated with silicone gel for insulation. A small nerve bundle is inserted into the center hole of the electrode (diameter, $0.15 \mathrm{~mm}$ ) through a slit. On the other hand, Fig. 1B illustrates the configuration of the water-absorbent polymer (WAP) electrode. The WAP electrode consists of a pair of Silastic silicone tubes (outer diameter, $0.625 \mathrm{~mm}$; 602-135, Dow Corning, Midland, MI, USA) bonded with silicon rubber. A small piece of WAP material (Kurarasoft, Kuraray, Osaka, Japan), to which a Teflon-coated silver wire (outer diameter, $0.1 \mathrm{~mm}$ ) is fixed, is inserted into the Silastic tubing. The width of the electrode is $1.7 \mathrm{~mm}$ and its height was $0.625 \mathrm{~mm}$ (Fig. 1B). The Silastic tubing has a center hole and an oblique slit. A small nerve bundle is inserted into the center hole of the electrode through the slit. Whem the WAP material absorbs body fluid, the swelling of the WAP results in making good contact with the nerve bundle. After a nerve is inserted into either the ECR or WAP electrode, the electrode-nerve complex is covered with silicone gel (932, Wacker Chemie, München, Germany) for insulation.

The electrical impedance of the ECR and WAP electrodes developed in this study was measured with a LCZ meter (ZM2355, NF Electric Instruments, Yokohama, Japan). As shown in Fig. 2, when the microelectrodes were embedded in saline, their electrical impedance was $2.8-9.4 \mathrm{k} \Omega$ in a frequency range of $100-5,000 \mathrm{~Hz}$, which approximately corresponds to the frequency band of sympathetic nerve discharge. There was no substantial difference in electrical impedance between the ECR and WAP electrodes. Both electrodes showed stable electrical impedance over several weeks. The results suggests that if either electrode has good contact with a nerve bundle, the impedance of the electrode is low enough to record sympathetic nerve activity with low background noise.

We manifested whether or not either implantable electrode could record renal sympathetic nerve activity (RSNA) in the anesthetized rat. Anesthesia was introduced by inhaling a gas mixture of $4 \%$ halothane 


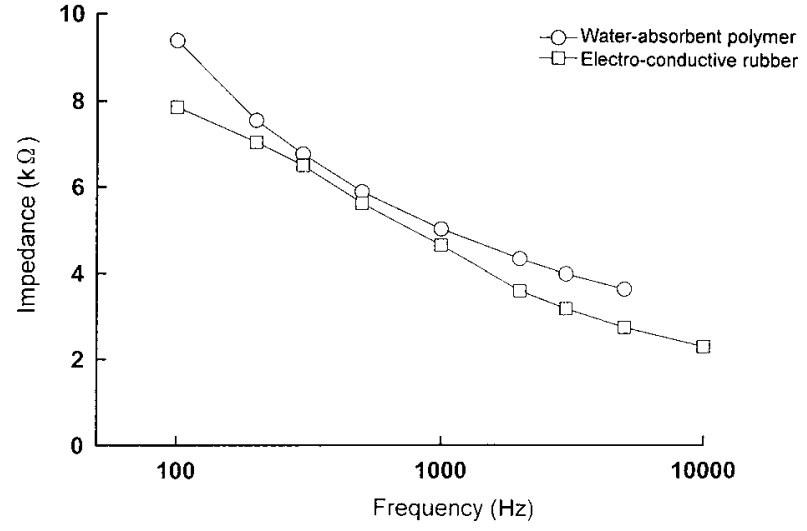

Fig. 2. Electrical impedances of electro-conductive rubber and water-absorbent polymer electrodes. When either microelectrode was embedded in saline, the impedance of the electrode was $2.8-9.4 \mathrm{k} \Omega$ in a frequency range of $100-5,000 \mathrm{~Hz}$, which approximately corresponds to the frequency band of sympathetic nerve discharge.

(Fluothane, Takeda Chemical Industries, Osaka, Japan), $\mathrm{N}_{2} \mathrm{O}$, and $\mathrm{O}_{2}$. Electrocardiogram (ECG), heart rate (HR), rectal temperature, and respiration were continuously monitored during surgery. After the left external jugular vein and carotid artery were cannuleted for administering drugs and measuring arterial blood pressure (AP), pentobarbital sodium (40-50 $\mathrm{mg} / \mathrm{kg}$ I.V.) was administered and gas anesthesia was stopped. Additional pentobarbital $(5-10 \mathrm{mg} / \mathrm{kg}$ I.V.) was injected if an increase in HR and/or respiration and/or withdrawal of the limb in response to noxious pinch of the paw and/or a surgical procedure was observed. Rectal temperature was maintained at 37.5$38.5^{\circ} \mathrm{C}$ with a heating pad. The left kidney was exposed and a renal nerve bundle was carefully isolated near the renal artery and vein. Either an ECR or WAP electrode was implanted on the nerve bundle, and the electrode-nerve complex was covered with silicone gel. The renal nerve was left intact. After the lead wires of the recording electrode were tunneled subcutaneously and brought to the exterior in the interscapular region, the incision was closed. As compared to a conventional electrode, the present cuff electrodes handled so well that it took only several minutes to insert the renal nerve into the electrode. In addition, since the ECR electrode was smaller than the WAP electrode and handled more easily, we feel that the ECR electrode may be preferable when implanting the electrode on a nerve bundle in a chronic study.

Original RSNA was amplified with a differential preamplifier (MEG-2100, Nihon Kohden, Tokyo, Japan). The amplified output was fed into a bandpass filter at between 50 and 3,000 Hz. Sympathetic discharges were converted into standard pulse trains

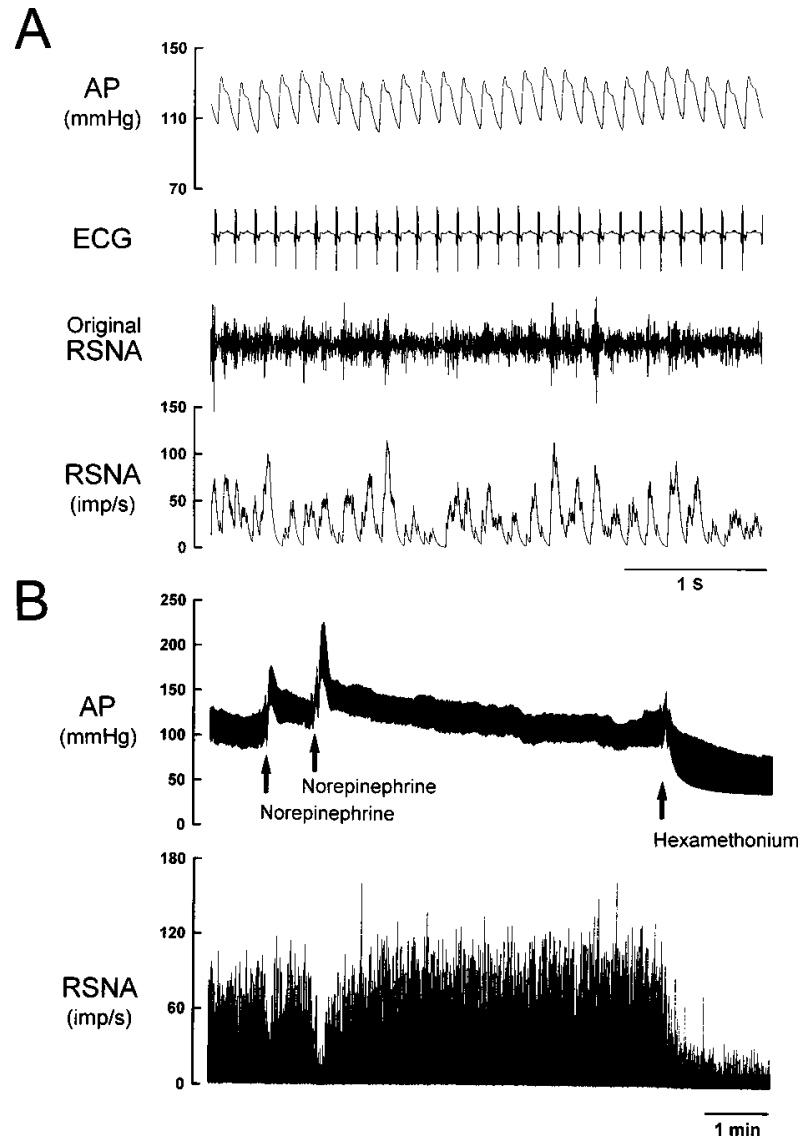

Fig. 3. An example of recording sympathetic discharge obtained from a renal nerve bundle in an anesthetized rat using an electro-conductive rubber electrode. RSNA, renal sympathetic nerve activity; $A P$, arterial blood pressure; ECG, electrocardiogram. In $\mathbf{A}$, a typical sympathetic discharge pattern of RSNA was seen. In B, RSNA was verified by intravenous injection of norepinephrine $(0.5-1 \mu \mathrm{g} / \mathrm{kg})$ or hexamethonium bromide (3 $\mathrm{mg} / \mathrm{kg})$.

using a digital technique that detected the peaks of the original neural spikes $[11,13,17]$. The pulse trains were integrated continuously with a resistance-capacitance integrator having a time constant of $20 \mathrm{~ms}$. The integrated signal was used as a monitor of RSNA. AP was measured through the carotid artery catheter connected to a pressure transducer (DPTIII, Baxter, Tokyo, Japan). Original RSNA, RSNA, AP, and ECG were stored in a computer with an analogue-digital converter (MP100, BIOPAC Systems, Santa Barbara, CA, USA) at a sampling frequency of $2,000 \mathrm{~Hz}$. Figure 3 demonstrates an example of RSNA with the ECR electrode. The RSNA showed characteristic grouped discharges synchronous with the cardiac cycle and respiratory movement. On the administration of norepinephrine $(0.5-1.0 \mu \mathrm{g} / \mathrm{kg}$ I.V.), RSNA was markedly reduced and the grouped nerve discharges almost disappeared. Intravenous administration of a ganglionic blocker (hexamethonium bro- 
mide, $3 \mathrm{mg} / \mathrm{kg}$ ) decreased RSNA remarkably as shown in Fig. 3. RSNA could be measured using either type of implantable electrode. As long as they are used in acute experiments, no substantial difference in the signal-to-noise ratio of sympathetic nerve activity between ECR and WAP electrodes is expected.

In conclusion, we have developed two novel types of bipolar cuff electrodes using electro-conductive rubber or water-absorbent polymer, either of which can be applied to the renal nerve in the rat and have good contact with the nerve bundle. Because the present novel electrodes are of small size and handle easily, it takes a shorter time to implant them on the nerve without nerve injury due to drying or mechanical damage, and both electrodes work well in acutetype experiments. The present novel electrodes may be useful for chronic recording of sympathetic nerve discharge in conscious animals. In particular, we feel that the ECR electrode, which is smaller and handles more easily, may be preferable for chronic implantation.

This study was supported by Grants-in-Aid for Scientific Research from the Ministry of Education, Culture, Sports, Science and Technology, Japan, and by "Ground-Based Research Announcement for Space Utilization" promoted by the Japan Space Forum.

\section{REFERENCES}

1. Ninomiya I, Yonezawa $Y$, and Wilson MF: Implantable electrode for recording nerve signals in awake animals. J Appl Physiol 41: 111-114, 1976

2. Schad $H$ and Seller $H$ : A method for recording autonomic nerve activity in unanesthetized, freely moving cats. Brain Res 100: 425-430, 1975

3. Matsukawa K and Ninomiya I: Changes in renal sympathetic nerve activity, heart rate and arterial blood pressure associated with eating in cats. J Physiol (Lond) 390: 229-242, 1987

4. Miki K, Kosho A, and Hayashida Y: Method for continuous measurements of renal sympathetic nerve activity and cardiovascular function during exercise in rats. Exp Physiol 87: 33-39, 2002

5. Matsukawa K, Mitchell JH, Wall PT, and Wilson LB: The effect of static exercise on renal sympathetic nerve ac- tivity in conscious cats. J Physiol (Lond) 434: 453-467, 1991

6. O'Hagan KP, Bell LB, Mittelstadt SW, and Clifford PS: Effect of dynamic exercise on renal sympathetic nerve activity in conscious rabbits. J Appl Physiol 74: 2099-2104, 1993

7. Dorward PK, Riedel W, Burke SL, Gipps J, and Korner PI: The renal sympathetic baroreflex in the rabbit. Arterial and cardiac baroreceptor influences, resetting, and effect of anesthesia. Circ Res 57: 618-633, 1985

8. Kunitake $\mathrm{T}$ and Kannan $\mathrm{H}$ : Discharge pattern of renal sympathetic nerve activity in the conscious rat: spectral analysis of integrated activity. J Neurophysiol 84: 2859-2867, 2000

9. Morita $\mathrm{H}$ and Vatner SF: Effects of hemorrhage on renal nerve activity in conscious dogs. Circ Res 57: 788-793, 1985

10. Miki K, Hayashida Y, Sagawa S, and Shiraki K: Renal sympathetic nerve activity and natriuresis during water immersion in conscious dogs. Am J Physiol 256: R299-R305, 1989

11. Ninomiya I, Matsukawa K, Honda T, Nishiura N, and Shirai M: Cardiac sympathetic nerve activity and heart rate during coronary occlusion in awake cats. Am J Physiol 251: H528-H537, 1986

12. Ninomiya I, Matsukawa K, and Nishiura N: Central and baroreflex control of sympathetic nerve activity to the heart and kidney in a daily life of the cat. Clin Exp Hypertens A 10 (Suppl 1): 19-31, 1988

13. Tsuchimochi $\mathrm{H}$, Matsukawa $\mathrm{K}$, Komine $\mathrm{H}$, and Murata $\mathrm{J}$ : Direct measurement of cardiac sympathetic efferent nerve activity during dynamic exercise. Am J Physiol Heart Circ Physiol 283: H1896-H1906, 2002

14. Jardine DL, Charles CJ, Melton IC, May CN, Forrester $\mathrm{MD}$, Frampton CM, Bennett SI, and Ikram H: Continual recordings of cardiac sympathetic nerve activity in conscious sheep. Am J Physiol Heart Circ Physiol 282: H93-H99, 2002

15. DiCarlo SE, Chen CY, and Collins HL: Onset of exercise increases lumbar sympathetic nerve activity in rats. Med Sci Sports Exerc 28: 677-684, 1996

16. Tsuchimochi $H$, Matsukawa $K$, Komine $H$, Murata J, Yonezawa Y, Kondo K, and Seki Y: New types of microelectrodes for recording sympathetic neural discharge. Jpn J Physiol 52 (Suppl): S77, 2002

17. Yonezawa Y, Ninomiya I, Nishiura N, and Kajita S: An analyzer for autonomic nerve activities in acute and chronic animals. IEEE Trans Biomed Eng 33: 63-66, 1986 